\title{
13. On Rings of Continuous Functions and the Dimension of Metric Spaces
}

\author{
By Jun-iti Nagata \\ Osaka City University and University of Washington \\ (Comm. by K. KUnUGi, M.J.A., Jan. 12, 1960)
}

M. Katětov [1] has once established an interesting theory on a relation between the inductive (Menger-Urysohn) dimension of a compact space $R$ and the structure of the ring of all continuous functions on $R$. The purpose of this brief note is to give a slight extension to Katětov's theory for a metric space while simplifying his discussion.

According to [1], we consider an analytical ring, i.e. a commutative topological ring with a unit $e$ and a continuous real scalar multiplication. A subring $C_{1}$ of an analytical ring $C$ is called analytically closed if

(1) $\lambda e \in C_{1}$ for any real $\lambda$, (2) $x \in C_{1}$ whenever $x \in C, x^{n}+a_{1} x^{n-1}+\cdots$ $+a_{n}=0, \quad a_{i} \in C_{1}, \quad$ (3) $\quad \bar{C}_{1}=C_{1}$.

Let $C^{\prime}$ be a subset of $C$; then a subset $M$ of $C$ is called an analytical base of $C^{\prime}$ in $C$ if there exists no analytically closed subring $C_{1} \doteq C^{\prime}$ containing $M$. The least number of an analytical base of $C^{\prime}$ in $C$ is called the analytical dimension of $C^{\prime}$ in $C$ and denoted by $\operatorname{dim}\left(C^{\prime}, C\right)$. The ring $C(R)$ of all bounded real-valued continuous functions of $R$ is an analytical ring as for its strong topology. We denote by $U(R)$ the subset of $C(R)$ consisting of all uniformly continuous functions. Furthermore, according to [2], we call a continuous mapping $f$ of a metric space $R$ into a metric space $S$ uniformly 0-dimensional if for any $\varepsilon>0$ there exists $\eta>0$ such that $\delta(U)<\varepsilon$ whenever $U \subset R$, diam $f(U)<\eta$, where $\delta(U)<\varepsilon$ means the fact that there exists an open covering $\mathfrak{B}$ of $U$ such that mesh $\mathfrak{B}=\sup \{\operatorname{diam} V \mid V \in \mathfrak{B}\}<\varepsilon$ and order $\mathfrak{B} \leqq 1$. The covering dimension of $R$ or the strong inductive dimension of $R$ as the same is denoted by $\operatorname{dim} R$. Now we can prove the following

Theorem. $\operatorname{dim} R=\operatorname{dim}(U(R), C(R))$ for every locally compact, metric space $R$.

To establish this theorem we prove some lemmas.

Lemma 1. Let $f(x)=\left(f_{1}(x), \cdots, f_{n}(x)\right)$ be a uniformly 0-dimensional, bounded mapping of a metric space $R$ into the $n$-dimensional Euclidean space $E_{n}$. Let $C_{1}$ be an analytically closed subring of $C(R)$ containing $f_{1}, \cdots f_{n}$; then for every sets $F$ and $G$ of $R$ with distance $(F, G)=d(F, G)>0$, there exists $g \in C_{1}$ such that $g(F) \geqq 1, g(G)=0$, where $g(F) \geqq 1$, for example, means that $g(x) \geqq 1$ for every $x \in F$. 
Proof. Let $d(F, G)=\varepsilon>0$ and take $\eta>0$ such that diam $f(U)<\eta$ for $U \subset R$ implies $\delta(U)<\varepsilon$. Choosing $\xi>0$ such that $\operatorname{diam} \prod_{i=1}^{n}\left(r_{i}-2 \xi\right.$, $\left.r_{i}+2 \xi\right)<\eta$ for every $r_{i}$, we cover $f(R)$ with finitely many cubes

Let

$$
I_{k}=\prod_{i=1}^{n}\left[r_{k i}-\xi, r_{k i}+\xi\right], \quad k=1, \cdots, l \text {. }
$$

where

$$
U_{k}=f^{-1}\left(I_{k}\right), \quad V_{k}=f^{-1}\left(J_{k}\right) \text {, }
$$

$$
J_{k}=\prod_{i=1}^{n}\left(r_{k i}-2 \xi, r_{k i}+2 \xi\right) \text {. }
$$

It easily follows from $f_{i} \in C_{1}$ that $f_{k i}=\left(2 \xi-\left|f_{i}-r_{k i}\right|\right) \frac{1}{\xi} \in C_{1}$, and hence $\bar{f}_{k}=\prod_{i=1}^{n} f_{k i} \in C_{1}$. Then

$$
\begin{array}{lll}
\bar{f}_{k}(x) \geqq 1 & \text { for every } & x \in U_{k}, \\
\bar{f}_{k}(x)>0 & \text { for every } & x \in V_{k}, \\
\bar{f}_{k}(x)=0 & \text { for every } & x \in \bar{V}_{k}-V_{k} .
\end{array}
$$

Since diam $f\left(V_{k}\right)=\operatorname{diam} J_{k}<\eta$, we can find an open covering $\mathfrak{V}_{k}$ of $V_{k}$ with mesh $\mathfrak{B}_{k}<\varepsilon$, order $\mathfrak{B}_{k} \leqq 1$. It is easy to see that $S\left(F, \mathfrak{V}_{k}\right)=W_{k}$

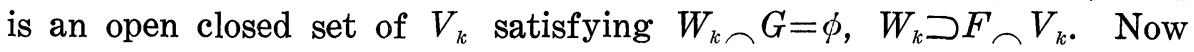
we define a function $g_{k}$ by

$$
\begin{array}{ll}
g_{k}(x)=\bar{f}_{k}(x), & x \in W_{k}, \\
g_{k}(x)=0, & x \notin W_{k} .
\end{array}
$$

Then since $g_{k}$ clearly satisfies $g_{k} \in C(R)$ and $g_{k}^{2}-\bar{f}_{k} g_{k}=0$ for $\bar{f}_{k} \in C_{1}$, we get $g_{k} \in C_{1}$ satisfying $g_{k}\left(F \frown U_{k}\right) \geqq 1, g_{k}(G)=0, g_{k} \geqq 0$. Letting $g=\sum_{k=1}^{l} g_{k}$ we have an element $g$ of $C_{1}$ satisfying $g(F) \geqq 1, g(G)=0, g \geqq 0$.

Lemma 2. $\operatorname{dim} R \geqq \operatorname{dim}(U(R), C(R))$ for every metric space $R$.

Proof. If $\operatorname{dim} R \leqq n$, then by [2] there exists a uniformly 0dimensional, bounded mapping $f(x)=\left(f_{1}(x), \cdots, f_{n}(x)\right)$ of $R$ into $E_{n}$. Hence any analytically closed subring $C_{1}$ of $C(R)$ containing $f_{1}, \cdots, f_{n}$ also contains, for every disjoint closed sets $F$ and $G$ with $d(F, G)>0$, $\varphi \in C_{1}$ such that $\varphi(F)=0, \varphi(G) \geqq 1$ by Lemma 2 . Hence by an analogous theorem to that of E. Hewitt [3, Theorem 1], we get, for every $\bar{\varphi} \in U(R)$ and $\varepsilon>0$ a polynomial $P\left(\varphi_{1}, \cdots, \varphi_{k}\right)$ in $\varphi_{i} \in C_{1}, i=1, \cdots, k$ such that $\left|\bar{\varphi}-P\left(\varphi_{1}, \cdots, \varphi_{n}\right)\right|<\varepsilon$. Therefore $\bar{\varphi} \in \bar{C}_{1}=C_{1}$, which implies $C_{1} \supseteqq U(R)$. Thus $\left(f_{1}, \cdots, f_{n}\right)$ is an analytical base of $U(R)$ in $C(R)$, i.e. $\operatorname{dim}(U(R)$, $C(R)) \leqq n$.

Lemma 3. $\operatorname{dim} R \leqq \operatorname{dim}(U(R), C(R))$ for every locally compact, metric space $R$.

Proof. Let $\left(f_{1}, \cdots, f_{n}\right)$ be an analytical base of $U(R)$ in $C(R)$; then $f(x)=\left(f_{1}(x), \cdots, f_{n}(x)\right)$ is a bounded continuous mapping of $R$ onto a subset $f(R)$ of $E_{n}$. Since $R$ is locally compact, there is a locally finite closed covering $\left\{R_{\alpha} \mid \alpha \in \Omega\right\}$ consisting of compact sets $R_{\alpha}$. Let $\mathfrak{U}$ be any finite open covering of $R_{\alpha}$; then there exists, for every $q \in f(R)$, 
a nbd (=neighborhood) $V(q)$ of $q$ in $f(R)$ such that $\delta\left(f^{-1} V(q)\right) \leqq \mathfrak{U}$, i.e. there exists an open covering $\mathfrak{B}$ of $f^{-1} V(q)$ satisfying $\mathfrak{B}<\mathfrak{U}$ in $R_{\alpha}$ and order $\mathfrak{B} \leqq 1$. It is enough to prove this proposition just for every binary open covering $\mathfrak{U}$ of $R_{\alpha}$. For we can find, for every finite open covering $\mathfrak{U}$ of $R_{\alpha}$, binary open coverings $\mathfrak{H}_{1}, \cdots, \mathfrak{U}_{k}$ of $R_{\alpha}$ satisfying $\mathfrak{H}_{1} \wedge \cdots \wedge \mathfrak{U}_{k}<\mathfrak{H}$. Then $\delta\left(f^{-1} V_{i}(q)\right) \leqq \mathfrak{U}_{i}, i=1, \cdots, k$ for $\operatorname{nbds} V_{i}(q), i=1$, $\cdots, k$ of $q$ imply $\delta\left(f^{-1} \stackrel{i=1}{\stackrel{h}{i=1}} V_{i}(q)\right) \leqq \mathbb{H}$. Now assume the contrary, i.e. let $F$ and $G$ be disjoint closed sets of $R_{\alpha}$ such that $\delta\left(f^{-1} V(q)\right) \leqq\left\{F^{c}, G^{c}\right\}$ for every $\operatorname{nbd} V(q)$ of $q$.

Let $D=\{g \mid g \in C(R)$, for every $\varepsilon>0$, there exist a nbd $V(q)$ of $q$ in $f(R)$ and an open covering $\mathfrak{U}$ of $f^{-1} V(q)$ such that mesh $g(\mathfrak{U})<\varepsilon$ and order $\mathfrak{U} \leqq 1\}$, where $g(\mathfrak{U})$ denotes the covering $\{g(U) \mid U \in \mathfrak{U}\}$ then $D$ is an analytically closed subring containing $f_{1}, \cdots, f_{n}$. Let us just show that $g \in D$ whenever $g \in C(R), g^{n}+a_{1} g^{n-1}+\cdots+a_{n}=0, a_{i} \in D$, where this $n$ is not related with the number of $f_{i}$. Let us denote by $g_{k}\left(b_{1}, \cdots, b_{n}\right)$, $k=1,2, \cdots, n$ the $n$ roots of the equation

$$
y^{n}+b_{1} y^{n-1}+\cdots+b_{n}=0 \text {. }
$$

Let $\left|a_{i}\right| \leqq K, i=1, \cdots, n$; then since $g_{k}\left(b_{1}, \cdots, b_{n}\right)$ are continuous functions of $b_{1}, \cdots, b_{n}$ and accordingly are uniformly continuous for $\left|b_{i}\right| \leqq K$, $i=1, \cdots, n$, for any $\varepsilon>0$ we can find $\delta>0$ such that

$$
\begin{gathered}
\left|b_{i}-b_{i}^{\prime}\right|<\delta,\left|b_{i}\right| \leqq K,\left|b_{i}^{\prime}\right| \leqq K, i=1, \cdots, n \text { imply } \\
\left|g_{k}\left(b_{1}, \cdots, b_{n}\right)-g_{k}\left(b_{1}^{\prime}, \cdots, b_{n}^{\prime}\right)\right|<\frac{\varepsilon}{n}, k=1, \cdots, n .
\end{gathered}
$$

Now let $V(q)$ be a nbd of $q$ and $\mathfrak{U}=\left\{U_{r} \mid \gamma \in \Gamma\right\}$ an open covering of $f^{-1} V(q)$ such that mesh $a_{i}(\mathfrak{U})<\delta, i=1, \cdots, n$ and order $\mathfrak{U} \leqq 1$. Moreover, let

$$
\begin{aligned}
& \left\{x \mid g_{k}\left(a_{1}(x), \cdots, a_{n}(x)\right)-g(x)=0, x \in U_{r}\right\}=U_{k r}, \\
& \left\{U_{k r} \mid k=1, \cdots, n\right\}=\mathfrak{H}_{r}, \\
& \left\{S^{n}\left(U_{k r}, \mathfrak{U}_{r}\right) \mid U_{k r} \in \mathfrak{U}_{r}\right\}=\mathfrak{B}_{r} .
\end{aligned}
$$

Then $\mathfrak{B}_{r}$ is an open covering of $U_{r}$ with order $\mathfrak{B}_{r} \leqq 1$ and mesh $g\left(\mathfrak{B}_{r}\right)$ $<\varepsilon$; hence $\mathfrak{B}=\smile\left\{\mathfrak{B}_{\gamma} \mid \gamma \in \Gamma\right\}$ is an open covering of $f^{-1} V(q)$ with order $\mathfrak{B} \leqq 1$ and mesh $g(\mathfrak{Z})<\varepsilon$. Thus we get $g \in D$. Since $R_{\alpha}$ is compact, it must be $d(F, G)>0$, and hence there exists a function $h \in U(R)$ such that $h(F)=0, h(G)=1$. However, from the assumption $D$ does not contain such a function $h$, which contradicts the fact that $\left(f_{1}, \cdots, f_{n}\right)$ is an analytical base of $U(R)$ in $C(R)$. Hence for every finite open covering $\mathfrak{U}$ of $R_{\alpha}$ and for every point $q$ of $f(R)$ there exists a nbd $V(q)$ of $q$ satisfying $\delta\left(f^{-1} V(q)\right) \leqq \mathfrak{U}$. Take an open refinement $\mathfrak{B}=\left\{V_{\gamma} \mid \gamma \in \Gamma\right\}$ of $\{V(q) \mid q \in f(R)\}$ with order $\mathfrak{B} \leqq n+1$. Then since $\delta\left(f^{-1}\left(V_{\gamma}\right)\right) \leqq \mathfrak{B}$, we can find an open covering $\mathfrak{W}_{r}$ of $f^{-1}\left(V_{r}\right)$ satisfying $\mathfrak{W}_{r}<\mathfrak{l l}$, order $\mathfrak{W}_{r} \leqq 1$. Now $\mathfrak{W}=\smile\left\{\mathfrak{W}_{r} \mid \gamma \in \Gamma\right\}$ restricted in $R_{\alpha}$ is an open refinement of $\mathfrak{U}$ with order $\mathfrak{W} \leqq n+1$. Therefore we can conclude $\operatorname{dim} R_{\alpha} \leqq n$. Hence, by 
use of the sum-theorem, we get $\operatorname{dim} R \leqq n$.

Combining Lemma 3 with Lemma 2 , we can conclude the validity of the theorem.

Incidentally, let us show the following

Corollary. $U(R)$ of every metric space $R$ has an analytical base in $C(R)$ consisting of countably many elements.

While checking up the proofs of Lemmas 1,2 , we know that this corollary is a direct consequence of the following

Lemma 4. Every metric space $R$ can be mapped into the Hilbert cube $I_{w}$ by a uniformly 0-dimensional mapping.

Proof. Since, by [4], every metric space $R$ can be imbedded in a product of countably many one-dimensional metric spaces, we can conclude this lemma from the fact owing to [2] that every one-dimensional metric space is mapped into $E_{1}$ by a uniformly 0 -dimensional, bounded function.

\section{References}

[1] M. Katětov: On rings of continuous functions and the dimension of compact spaces, Časopis pro pěstování matematiky a fysiky, 75, 1-16 (1950).

[2] - : On the dimension of non-separable spaces I, Czechoslovak Mathematical Jour., 2 (77), 333-368 (1952).

[3] E. Hewitt: Certain generalization of the Weierstrass approximation theorem, Duke Math. Jour., 14, 419-427 (1947).

[4] J. Nagata: On imbedding a metric space in a product of one-dimensional spaces, Proc. Japan Acad., 33, 445-449 (1957). 